\title{
The impact of vancomycin trough concentrations on outcomes in non-deep seated infections: a retrospective cohort study
}

Michael Wan ${ }^{1}$, Sandra A. N. Walker ${ }^{1,2,3,4^{*}}$ D, Elaine Martin ${ }^{7}$, Marion Elligsen ${ }^{1}$, Lesley Palmay ${ }^{1}$ and Jerome A. Leis $3,4,5,6$

\begin{abstract}
Background: Guidelines recommending vancomycin trough concentrations $>10 \mathrm{mg} / \mathrm{L}$ in non-deep seated infections are based on expert opinion. The objective of this study was to evaluate patients with non-deep seated infections treated with short-course vancomycin to determine whether there were differences in outcomes with trough concentrations of $\leq 10 \mathrm{mg} / \mathrm{L}$ (low) versus $>10 \mathrm{mg} / \mathrm{L}$ (high).

Methods: A retrospective cohort study of patients hospitalized between March 10, 2010 and December 31, 2015 who received $\leq 14$ days of vancomycin to treat a non-deep seated infection and had at least one steady state trough concentration was completed. Patient data for the low versus high trough cohorts were compared using appropriate statistical tests and binary logistic regression was used to identify factors associated with clinical outcome.

Results: Of 2098 patients screened, 103 (5\%) met inclusion criteria. Baseline characteristics between cohorts were not different. Clinical cure was not different between the low (42/48 [88\%]) and high trough (48/55 [87\%]) cohorts $(p>0.99)$ and vancomycin trough concentration was not associated with clinical outcome $(p=0.973)$. More patients in the high trough group had dosing changes (7/48 [15\%] vs. $22 / 55$ [40\%], $p=0.0046)$, with approximately three times more dose adjustments per patient ( 0.17 vs. $0.55, p=0.0193$ ). No signal for increased vancomycin resistance associated with vancomycin troughs was identified.

Conclusions:: No difference in clinical or microbiological outcomes based on vancomycin trough concentrations were observed in patients with non-deep seated infections treated with vancomycin for $\leq 14$ days. Targeting higher vancomycin trough concentrations of $>10 \mathrm{mg} / \mathrm{L}$ may be associated with increased workload with no corresponding benefit in clinical or microbiological outcomes in these patients.
\end{abstract}

Keywords: Vancomycin, Non-deep seated infections, Trough concentrations, Levels, Therapeutic drug monitoring, Outcomes

\footnotetext{
* Correspondence: sandra.walker@sunnybrook.ca

'Department of Pharmacy, Sunnybrook Health Sciences Centre, 2075

Bayview Avenue, Toronto, ON M4N 3M5, Canada

${ }^{2}$ Leslie Dan Faculty of Pharmacy, University of Toronto, 144 College Street,

Toronto, ON M5S 3M2, Canada

Full list of author information is available at the end of the article
}

(c) The Author(s). 2018 Open Access This article is distributed under the terms of the Creative Commons Attribution 4.0 International License (http://creativecommons.org/licenses/by/4.0/), which permits unrestricted use, distribution, and reproduction in any medium, provided you give appropriate credit to the original author(s) and the source, provide a link to the Creative Commons license, and indicate if changes were made. The Creative Commons Public Domain Dedication waiver (http://creativecommons.org/publicdomain/zero/1.0/) applies to the data made available in this article, unless otherwise stated. 


\section{Background}

Vancomycin was discovered over 60 years ago; however, it was not until the early 1980s that its clinical use sharply increased in response to a rise in the worldwide prevalence of methicillin-resistant Staphylococcus aureus (MRSA) [1]. Unfortunately, controversy regarding the optimal dosing and pharmacokinetic/pharmacodynamic targets continues to plague the use of vancomycin [2]. Current guidelines recommend targeting vancomycin serum trough concentrations of $15-20 \mathrm{mg} / \mathrm{L}$ for complicated infections (e.g. endocarditis, osteomyelitis, pneumonia, meningitis, etc.), and suggest maintaining a trough of $>10 \mathrm{mg} / \mathrm{L}$ for all patients (including those with non-deep seated infections with a planned duration of $\leq 14$ days) to avoid development of resistance to vancomycin (Quality and Grade of Evidence for each recommendation in the 2009 IDSA guidelines was IIIB) [3-5].

The emergence of vancomycin resistance has not been observed in patients treated with short term vancomycin ( $\leq 14$ days) for the management of non-deep seated infections (e.g. bacteremia when endocarditis or other non-cardiac seeding [e.g. bone or brain] has been ruled out; uncomplicated skin and soft tissue infections; and urinary tract infections in the absence of anatomic abnormalities, renal stones, or renal abscess).

Clinical evidence to support the need to maintain vancomycin serum trough concentrations above $10 \mathrm{mg} / \mathrm{L}$ for management of non-deep seated infections is lacking and the practice of targeting higher trough concentrations has obvious downsides. This practice increases the complexity of the dosing and monitoring of vancomycin, results in additional blood draws from the patient, increases the workload for the clinical team, and may therefore, increase the risk of medical error and harm to the patient. We hypothesize that there is no difference in clinical or microbiological outcomes associated with vancomycin trough concentrations of $\leq 10 \mathrm{mg} / \mathrm{L}$ versus $>10 \mathrm{mg} / \mathrm{L}$ when vancomycin is used in the setting of non-deep seated infections for a period of $\leq 14$ days.

The primary objective of this study was to evaluate patients with non-deep seated infections who were treated with vancomycin for a period of $\leq 14$ days to determine whether there were differences in clinical outcome with serum trough concentrations of vancomycin of $\leq 10 \mathrm{mg} / \mathrm{L}$ versus $>10 \mathrm{mg} / \mathrm{L}$. The secondary objectives of this study were to identify factors which may affect clinical outcome, evaluate safety outcomes (kidney injury), assess workload based on dose adjustment(s), and identify changes in microbial resistance during the vancomycin treatment course.

\section{Methods}

\section{Study design}

A retrospective chart review of eligible patients was conducted. Patients were identified for eligibility using the
Stewardship Program Integrating Resource Information Technology (SPIRIT) database of our Antimicrobial Stewardship Program [6]. A SPIRIT query generated a list of inpatients at SHSC between March 12th, 2010 and December 31st, 2015, inclusive, with at least one steady state vancomycin trough concentration. Hospital charts for the patients identified by this query were retrieved from Health Data Resources (HDR) and reviewed to confirm patient eligibility for this study.

\section{Patient eligibility}

Adult inpatients (aged $\geq 18$ years) were eligible for study inclusion if they were admitted to SHSC between March 12th, 2010 and December 31st, 2015, received a minimum of $48 \mathrm{~h}$ and a maximum of 14 days of vancomycin for a presumed or confirmed non-deep seated infection (defined below) associated with any bacteria for which vancomycin may be indicated, and for whom at least one steady state vancomycin trough concentration was available. A steady state vancomycin trough concentration was defined as a level obtained at the earliest prior to the 3rd dose for $\geq$ every $12 \mathrm{~h}$ dosing, and prior to the 4th dose for $\leq$ every $8 \mathrm{~h}$ dosing; or a random vancomycin concentration obtained at least $24 \mathrm{~h}$ after vancomycin initiation in patients receiving continuous infusion. An exception was made for inclusion of patients receiving a maximum of 15 days of vancomycin, if the treating physician's intent was to treat for 14 days. Patients were eligible for inclusion if their infection-related diagnosis was:

- Uncomplicated skin and soft tissue infections (SSTI): folliculitis, carbuncle(s), surgical site infections, wound infections, non-suppurative cellulitis or erysipelas;

- Urinary tract infections (UTI) in the absence of anatomic abnormalities, renal abscess, or renal stones;

- Bacteremia without any evidence of seeding to heart, bone/joint, brain, or lungs; or

- Coagulase negative Staphylococci (CNST) linerelated infections (excluding tunnel infection or vascular graft infections).

If patients received more than one course of vancomycin during their hospital stay, only data from their first vancomycin course was included, with documentation of any subsequent courses of vancomycin within 14 days of vancomycin discontinuation or patient readmission within 30 days of completion of their initial vancomycin treatment course. Subsequent vancomycin treatment courses and patient readmission were considered markers of potential relapsing infection (clinical failure) with a risk of development of a vancomycin resistant bacterial isolate. Patients were excluded if there 
was a presumed or documented diagnosis of abscess at any site, endocarditis, meningitis, osteomyelitis, joint infection, febrile neutropenia, pneumonia, or sinusitis; were switched to an alternate antibiotic on day 2 or 3 of vancomycin due to culture and sensitivity results; received antibiotics for no documented infection (e.g. prophylactic antibiotics); received renal replacement therapy; or if vancomycin was discontinued as a result of a patient care plan that was changed to palliative.

\section{Data collection and definitions}

Required patient clinical and laboratory data were extracted from the hospital's electronic databases (SPIRIT, electronic patient records [EPR/Sunnycare]) and patient medical records (HDR/Sovera) and entered into a Microsoft Excel workbook. Patients were stratified to vancomycin low or high trough cohorts based on their final documented vancomycin steady-state trough concentration $(\leq 10 \mathrm{mg} / \mathrm{L}$ or $>10 \mathrm{mg} / \mathrm{L}$, respectively). The primary outcome of this study was clinical cure, defined as all of the following:

- Resolution of all presenting signs and symptoms of infection within $\leq 14$ days therapy with vancomycin; whereby patients could be discharged home on a short course of vancomycin (total duration $\leq 14$ days) with documentation of improvement during hospital stay;

- Maintained resolution of all presenting signs and symptoms of infection for 14 days following vancomycin discontinuation;

- No additional course of antibiotics within 14 days with the same indication as initial vancomycin treatment course;

- No documentation of hospital readmission requiring antibiotic therapy within 30 days of completion of their initial vancomycin treatment course.

Secondary outcomes:

- Identification of clinical, demographic or microbiological factors associated with clinical cure;

- Kidney injury outcomes as per RIFLE (Risk, Injury, Failure, Loss of kidney function, and End-stage kidney disease) criteria [7];

- Workload based on number of dose adjustment(s) during the vancomycin course; and

- Development of microbiological resistance during the vancomycin treatment course or within 30 days of completion of the initial vancomycin treatment course.

Severity of illness was measured with the Acute Physiology and Chronic Health Evaluation (APACHE) II score for intensive care unit (ICU) patients and Pitt Bacteremia score for ward patients $[8,9]$. As there are no other validated measures of severity of illness in hospital ward patients, the Pitt Bacteremia score was used in these patients, recognizing that the Pitt Bacteremia score has only been validated in patients with bacteremia. Hospital location (ICU versus ward) at time of vancomycin initiation was identified and ICU patients included those with admission or transfer to a critical care bed within $48 \mathrm{~h}$ of vancomycin initiation. Concomitant antibiotics were defined as any antibiotic whose administration overlapped that of vancomycin by at least $48 \mathrm{~h}$ and was administered for at least $48 \mathrm{~h}$. The time required for each vancomycin dose adjustment was estimated from typical nursing, physician, pharmacist and pharmacy technician practices at our institution (Appendix).

\section{Sample size calculation}

The literature was reviewed to provide an estimate of the vancomycin treatment failure rate in non-deep seated infections. Although three studies provided a treatment failure rate in SSTIs (ranging from 12 to 38\% for all organisms; and $17-35 \%$ for MRSA only), the SSTIs included in these studies were complicated skin and soft tissue infections (cSSTIs) that involved deep soft tissue or required significant surgical intervention (infected ulcers, burns, and major abscesses) [10-12]. No reliable treatment failure estimates were available for UTIs or isolated uncomplicated bacteremia associated with bacteria for which vancomycin may be used. Since only patients with non-deep seated infection(s) were eligible for this study, the treatment failure rates were predicted to be lower than those reported in the literature for cSSTIs.

Based on the only available published literature, we estimated our rate of treatment failure to be between 10 and $25 \% ; 10 \%$ being a hypothesized estimate, and $25 \%$ being the midpoint of treatment failure rates observed in the literature for cSSTIs. Thus, using a standard sample size equation for dichotomous data, 93 to 348 patients per group (based upon treatment failure rates of 10 to $25 \%$ in the high trough cohort) would be required to detect a minimal difference in failure $(\Delta)$ of 10 percentage points between patients with a trough of $\leq 10 \mathrm{mg} / \mathrm{L}$ versus $>10 \mathrm{mg} / \mathrm{L}$ (2-tailed, $p=0.05$, power $=0.80)$.

\section{Statistics \& data analysis}

Comparison of the study cohorts for interval, nominal and ordinal data were analyzed using GraphPad InStat (version 3.05, 32-bit for Win95/NT; GraphPad Software Inc., La Jolla, California). Nominal data were expressed as total number (proportion) of patients; and low trough and high trough groups were compared using the Fisher Exact test (odds ratio, 95\% confidence interval and 
$p$-value). Interval data were expressed as mean \pm standard deviation (and range) and the Kolmogorov Smirov test was used to evaluate the data for normality. If interval data passed the test for normality and standard deviations were not significantly different then a two-sided unpaired t-test was used to compare the study cohorts. If interval data were normally distributed, but standard deviations between groups were significantly different then the cohorts were compared using the two-sided unpaired t-test with Welch correction. If interval data did not pass the test for normality then a two tailed Mann-Whitney test was used. A $p$-value of $<0.05$ was considered as statistically significant.

A Pearson's Correlation matrix (univariable analysis) (SPSS version 13.0 for Windows, created September 1, 2004) was completed to identify patient clinical, microbiological, laboratory and vancomycin dosing related parameters (independent variables) associated with clinical cure (dependent variable). Patient related parameters that were evaluated by univariable analysis as independent variables were: sex, age, hospital location, length of stay at vancomycin initiation, comorbidities, immunosuppressive medications, nephrotoxic medications, severity of illness, baseline serum creatinine, final serum creatinine, serum creatinine change, risk of renal injury, kidney injury, Gram positive bacterial species identified in culture, and infectious diagnosis for which vancomycin was prescribed. Vancomycin dosing related factors that were evaluated by univariable analysis were initial dose, final dose, initial steady state vancomycin trough, final steady state vancomycin trough, and high versus low trough cohort. Any independent variables that were available for $>20 \%$ of patients and had a $p$ value $<0.05$ with Pearson's Correlation were maintained in the multivariable analysis of binary logistic regression (SPSS version 13.0 for Windows, created September 1, 2004) to identify the existence of a statistically significant model $(p<$ 0.05 ) in which all independent variables remaining in the model had an odds ratio of $>1$.

\section{Results}

A total of 2098 patients on vancomycin during the study period were identified from the SPIRIT database query for screening, and 103 (5\%) met inclusion criteria for the study (Low trough cohort: $n=48$, High trough cohort: $n$ =55) (Fig. 1). Of the 1995 excluded patients, the most common reason for exclusion was the presence of a deep-seated infection (1134 patients). The two groups were well-balanced in terms of baseline demographic, clinical and microbiological factors (Table 1).

There was no significant difference in clinical cure ( $88 \%$ vs. $87 \%$, OR $1.02,95 \%$ CI $0.32-3.28, p>0.99$ ) or survival $(100 \%$ vs. $98 \%$, OR $2.67,95 \%$ CI $0.11-67.13, p>$ $0.99)$ between the low trough and high trough groups (Table 2). One patient passed away in the high trough group for a reason unrelated to infection. Two patients in the high trough group (4\%) and no patients in the low trough group $(0 \%)$ were re-admitted within 30 days for the same indication as the original course of vancomycin. The 95\% confidence intervals around clinical cure in the low trough versus high trough cohorts were $78-97 \%$ versus $78-96 \%$, which completely overlap to support the finding of no significant difference in clinical cure. To detect a minimum difference in treatment failure of $1 \%$, a sample size of 17,370 patients per group would be required. To detect a minimum difference in treatment failure of $0.5 \%$, a sample size of $68,850 \mathrm{pa}$ tients per group would be required. A Monte Carlo Simulation (Oracle Crystal Ball, build 11.1.4100 on 12/ 23/2014, 32-bit) (MCS) of 1 million iterations, using a binomial distribution, with the study observed probability of cure (Low trough $=0.88$, High trough $=0.87$ ), was conducted to determine the probability that clinical cure could theoretically be $\geq 10 \%$ better in the high trough cohort compared to the low trough cohort. This probability was determined to be $3 \%$.

The average final steady state vancomycin troughs were $7.38 \pm 1.95 \mathrm{mg} / \mathrm{L}$ and $16.56 \pm 6.56 \mathrm{mg} / \mathrm{L}$ in the low and high trough groups, respectively, and the median duration of therapy in both groups was $7 \pm 3$ days. Although the median total daily dose between groups was similar ( $2 \mathrm{~g}$ per day in both groups), patients in the high trough group were more likely to receive 3 or more grams of vancomycin per day $(p=0.04)$ and had a significant increase from initial to final vancomycin troughs $(p=0.0009)$. Patients in the high trough group had more vancomycin dosing changes $(p=0.02)$, which in turn translated to a three-fold higher investment of health care worker time related to vancomycin therapy per patient in the high trough group $(\mathrm{p}=0.02)$ (Table 2). Renal function outcomes were similar between the two groups, with no significant difference in change in serum creatinine $(p=0.13)$ and number of patients at risk for kidney injury $(p=0.12)$. However, it is noteworthy that while no patient in the low trough group was at risk for kidney injury, 4 patients in the high trough group were at risk for kidney injury (Table 2).

Univariable analysis identified five independent variables that were significantly associated with clinical outcome; however, none of these variables remained significant with multivariable analysis (Table 3). Notably, low/high trough categorization was not significantly associated with clinical outcome in patients with a non-deep seated infection with univariable analysis. The patient to variable ratio for the multivariable analysis was 21:1.

\section{Discussion}

This retrospective study evaluated whether differences in clinical cure exist when targeting trough concentrations 


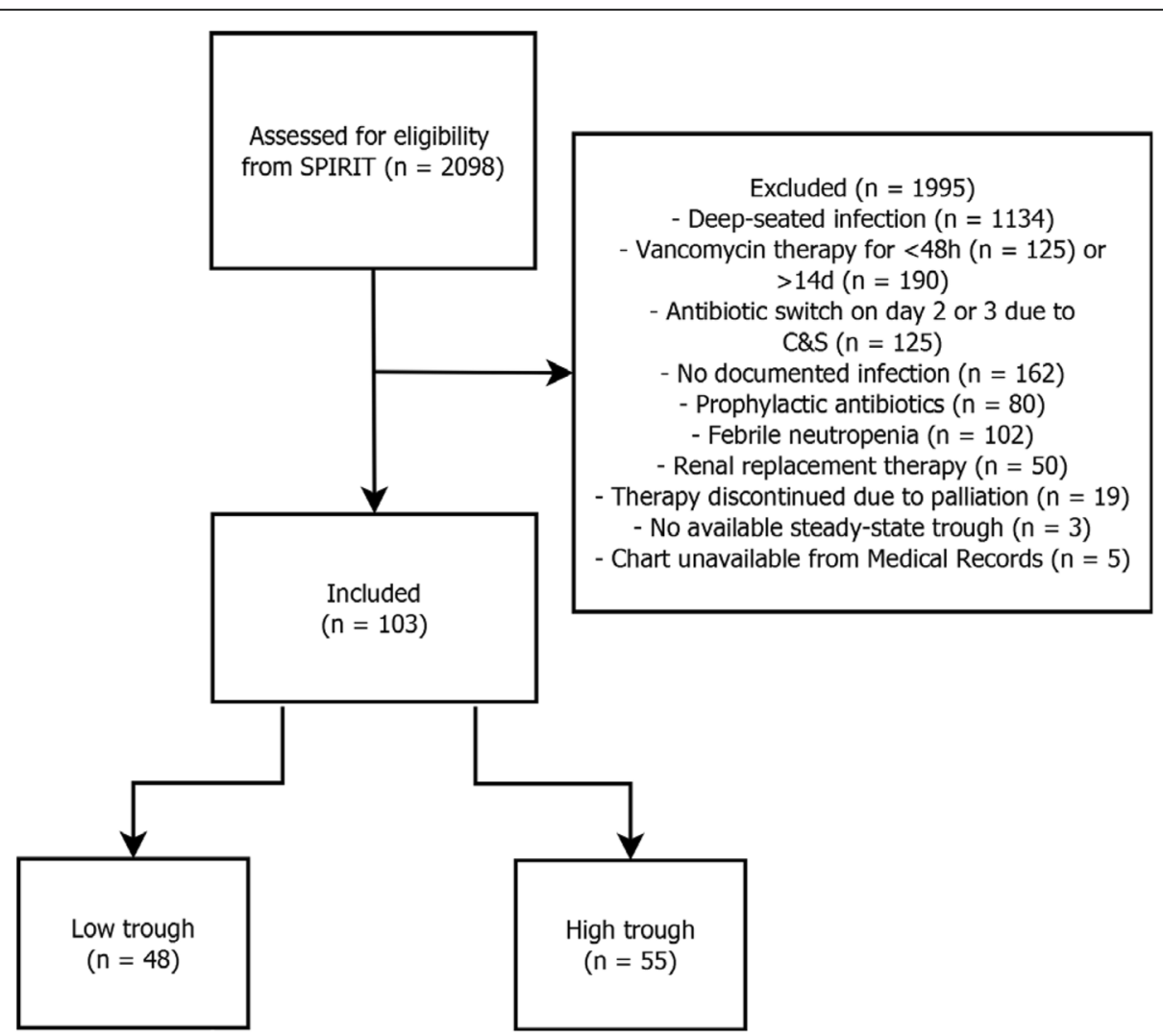

Fig. 1 Inclusion and exclusion of flow diagram

$\leq 10 \mathrm{mg} / \mathrm{L}$ versus $>10 \mathrm{mg} / \mathrm{L}$ in patients with non-deep seated infections treated with vancomycin for $\leq 14$ days; and, to the best of our knowledge, is the first study to do so. Although limited by a small sample size, the study enrollment reflects the entire population of patients with non-deep seated infections treated with vancomycin at our hospital between March 12th, 2010 and December 31st, 2015, inclusive. Strict eligibility criteria for study entry were used and the cohorts were well balanced for all baseline characteristics. We did not measure any difference in clinical cure with higher vancomycin trough concentrations and there was no signal for selection of resistant Gram positive bacteria in patients with a non-deep seated infection receiving $\leq 14$ days of vancomycin. However, there was a three-fold increase in healthcare personnel workload, which introduces unnecessary complexity (increased pharmacist, pharmacy technician, nursing and physician time involvement, additional blood work, and risk of medication error with more frequent dose adjustments).

In current infectious diseases guidelines, the rationale for maintaining trough concentrations above $10 \mathrm{mg} / \mathrm{L}$ is to prevent the development of resistance; emergence of vancomycin resistance in patients treated with $\leq 14$ days of vancomycin has never been reported in the literature [3-5].
We did not observe the emergence of any resistance to vancomycin in our study, and no patients with low vancomycin levels $(<10 \mathrm{mg} / \mathrm{L})$ required re-admission for recurrent infection; this is a reassuring finding, since one reason for recurrent infection may be the emergence of vancomycin resistance. Vancomycin resistance in Staphylococcus aureus with low vancomycin levels for $\leq 14$ days has only been observed in vitro with purposeful selection; and clinically in patients who received prolonged vancomycin exposure (6-18 weeks) [2, 13-23]. No association between vancomycin trough concentrations and emergence of vancomycin resistance has been observed for any other bacteria (e.g. CNST, Enterococci, other Gram positive organisms) for which vancomycin may be indicated.

As a single-center, retrospective analysis, our study has several limitations. To maximize our sample size, we screened all potential patients since the inception of our Antimicrobial Stewardship database until study closing. Despite our efforts, our final sample size was only sufficient to detect a difference of $25 \%$ points between the two groups for our primary outcome; we set a difference of $10 \%$ between the two groups as being clinically important to identify in our sample size calculation, and observed a difference of less than $1 \%$ (non-rounded clinical cure rates in low versus high trough cohorts $87.5 \%$ vs. $87.3 \%$ ) between 
Table 1 Patient Characteristics $(N=103)$

\begin{tabular}{|c|c|c|c|c|c|}
\hline & Low Trough $(N=48)$ & $\begin{array}{l}\text { High Trough } \\
(N=55)\end{array}$ & Odds Ratio & 95\% Confidence Interval & $p$-value \\
\hline Gender (Male) & $27(56 \%)$ & $28(51 \%)$ & 1.24 & $0.57-2.70$ & 0.69 \\
\hline Age on Admission (Years), mean \pm SD (Range) & $59 \pm 19(21-91)$ & $67 \pm 21(19-96)$ & & & 0.06 \\
\hline Hospital Location at Vancomycin Initiation (Ward) & $38(79 \%)$ & $44(80 \%)$ & 0.95 & $0.36-2.48$ & $>0.99$ \\
\hline $\begin{array}{l}\text { Length of Stay at the time of Vancomycin Initiation } \\
\text { (Days),median'(Range) }\end{array}$ & $2(0-70)$ & $2(0-187)$ & & & 0.89 \\
\hline Any Comorbidity ${ }^{b}$ & $19(40 \%)$ & $25(45 \%)$ & 0.79 & $0.36-1.72$ & 0.56 \\
\hline - Congestive Heart Failure & $3(6 \%)$ & $8(15 \%)$ & 0.39 & $0.098-1.57$ & 0.21 \\
\hline - Chronic Obstructive Pulmonary Disease & $2(4 \%)$ & $7(13 \%)$ & 0.30 & $0.06-1.51$ & 0.17 \\
\hline - Diabetes Mellitus & $7(15 \%)$ & $10(18 \%)$ & 0.77 & $0.27-2.21$ & 0.79 \\
\hline - Immunosuppression due to disease or $\mathrm{drug}^{c}$ & $8(17 \%)$ & $6(11 \%)$ & 1.63 & $0.52-5.10$ & 0.57 \\
\hline APACHE $\|^{d}$, mean \pm SD (ICU patients) & $17 \pm 6(11-25)$ & $23 \pm 11(6-41)$ & & & 0.32 \\
\hline $\begin{array}{l}\text { Critically III Ward Patients } \\
\text { (Pitt Bacteremia Score } \geq 4 \text { ) }\end{array}$ & $1(2 \%)$ & $4(7 \%)$ & 0.27 & $0.03-2.52$ & 0.37 \\
\hline Baseline Creatinine (mmol/L) & $77 \pm 32(23-185)$ & $83 \pm 45(18-254)$ & & & 0.42 \\
\hline Use of Concomitant Nephrotoxins ${ }^{e}$ & $35(73 \%)$ & $36(65 \%)$ & 1.42 & $0.61-3.31$ & 0.52 \\
\hline - \# of Concomitant Nephrotoxins ${ }^{\mathrm{a}}$ (median) & $1(0-3)$ & $1(0-4)$ & & & 0.49 \\
\hline Use of Concomitant Antibiotics ${ }^{f}$ & $21(44 \%)$ & $17(31 \%)$ & 1.74 & $0.78-3.90$ & 0.22 \\
\hline - Same Indication as Vancomycin ${ }^{9}$ & $18(86 \%)$ & $14(82 \%)$ & 1.29 & $0.22-7.37$ & $>0.99$ \\
\hline \multicolumn{6}{|l|}{ Included Infections } \\
\hline - All SSTIS & $31(65 \%)$ & $32(58 \%)$ & 1.31 & $0.59-2.91$ & 0.55 \\
\hline o Cellulitis & $25(52 \%)$ & $22(40 \%)$ & 1.63 & $0.75-3.57$ & 0.24 \\
\hline o Wound/Surgical Site Infection & $6(12 \%)$ & $10(18 \%)$ & 0.64 & $0.21-1.92$ & 0.59 \\
\hline - All UTIs & $11(23 \%)$ & $7(13 \%)$ & 2.04 & $0.72-5.77$ & 0.20 \\
\hline O MRSA UTI & $2(4 \%)$ & $1(2 \%)$ & 2.35 & $0.21-26.75$ & 0.60 \\
\hline o Enteroccocal UTI & $9(19 \%)$ & $6(11 \%)$ & 1.88 & $0.62-5.75$ & 0.28 \\
\hline - All Bacteremias & $4(8 \%)$ & $10(18 \%)$ & 0.41 & $0.12-1.40$ & 0.16 \\
\hline o CNST Bacteremia ${ }^{\text {h }}$ & $3(6 \%)$ & $8(15 \%)$ & 0.39 & $0.10-1.57$ & 0.21 \\
\hline - Any Positive CNST in Blood & $5(10 \%)$ & $14(25 \%)$ & 0.34 & $0.11-1.03$ & 0.07 \\
\hline \multicolumn{6}{|l|}{ Microbiology } \\
\hline $\begin{array}{l}\text { - Patients With Positive Non-Screening Cultures } \\
\text { for Resistant Gram positive isolates' }\end{array}$ & $10(21 \%)$ & $22(40 \%)$ & 0.39 & $0.16-0.95$ & 0.054 \\
\hline o Patients with MRSA Clinical Culture & $2(4 \%)$ & $5(9 \%)$ & 0.43 & $0.08-2.35$ & 0.44 \\
\hline o Patients with CNST Clinical Culture & $8(17 \%)$ & $17(31 \%)$ & 0.45 & $0.17-1.16$ & 0.11 \\
\hline - MRSA-colonized Patients & $2(4 \%)$ & $8(15 \%)$ & 0.26 & $0.05-1.27$ & 0.10 \\
\hline - VRE-colonized Patients & $1(2 \%)$ & 0 & 3.50 & $0.14-88.15$ & 0.47 \\
\hline
\end{tabular}

ACEI Angiotensin converting enzyme inhibitor, AIDS Acquired immune deficiency syndrome, APACHE Acute Physiology and Chronic Health Evaluation, ARB Angiotensin II receptor blocker, ASA Acetylsalicylic acid, HCTZ Hydrochlorothiazide, CNST Coagulase-negative Staphylococci, HIV Human immunodeficiency virus, ICU Intensive Care Unit, NSAID Nonsteroidal anti-inflammatory drug, PPI Proton pump inhibitor, SD Standard deviation, SSTI Skin and soft tissue infection, TNF- $a$ Tumor necrosis factor alpha, UTI Urinary tract infection, VRE Vancomycin-resistant Enterococci

${ }^{a}$ For non-normally distributed data, the median with range was reported

batients may have had more than 1 comorbidity, thus totals for specific comorbidities sum to a value greater than the number of patients with any comorbidity

'Disease: HIV/AIDS, asplenia, hematological malignancies, transplantation. Drug: Corticosteroids (prednisone $>5 \mathrm{mg} /$ day, chemotherapy, TNF-a inhibitors, transplant medications)

${ }^{\mathrm{d}}$ Arterial blood gases were not available for all ICU patients and APACHE II scoring could not be completed for these patients; reported values are based on 6 patients in low trough cohort and 7 patients in high trough cohort

'NSAIDs, ACEls, ARBs, cyclosporine, tacrolimus, acyclovir, aminoglycosides, amphotericin, colistin, indinavir, adefovir, cidofovir, tenofovir, chemotherapy (e.g. carmustine, semustine, cisplatin, methotrexate, mitomycin), foscarnet, contrast dye, zoledronate, loop diuretics, HCTZ, triamterene, hydralazine, interferons, PPIs, sulfonamides, lithium, aristocholic acid, acetaminophen at $>1 \mathrm{~g} /$ day for $>2$ years, ASA at $>1 \mathrm{~g} /$ day for $>2$ years)

fConcomitant antibiotic defined as: $\geq 48 \mathrm{~h}$ overlap with vancomycin and administered for $\geq 48 \mathrm{~h}$

${ }^{9}$ Denominator for percentage calculations is the number of patients (n) on concomitant antibiotics (i.e. low trough $n=18$; high trough $n=14$ )

${ }^{\mathrm{h}}$ Defined as $\geq 2$ positive blood cultures on the same day

'Methicillin Resistant Staphylococcus aureus, Coagulase-negative Staphylococci, or Vancomycin Resistant Enterococci cultured from 3 days prior to initiation or during vancomycin course of therapy and includes a single positive culture for CNST 
Table 2 Results $(N=103)$

\begin{tabular}{|c|c|c|c|c|c|}
\hline & $\begin{array}{l}\text { Low Trough } \\
(N=48)\end{array}$ & $\begin{array}{l}\text { High Trough } \\
(N=55)\end{array}$ & Odds Ratio & $\begin{array}{l}95 \% \text { Confidence } \\
\text { Interval }\end{array}$ & $p$-value \\
\hline \multicolumn{6}{|l|}{ Clinical Outcomes } \\
\hline • Clinical Cure (Primary Outcome) & $42(88 \%)$ & $48(87 \%)$ & 1.02 & $0.32-3.28$ & $>0.99$ \\
\hline - Survival & $48(100 \%)$ & $54(98 \%)$ & 2.67 & $0.11-67.13$ & $>0.99$ \\
\hline $\begin{array}{l}\text { - Readmission within } 30 \text { days for same indication as original } \\
\text { course of vancomycin }\end{array}$ & $0(0 \%)$ & $2(4 \%)$ & 0.22 & $0.01-4.71$ & 0.50 \\
\hline \multicolumn{6}{|l|}{ Microbiological Outcome } \\
\hline $\begin{array}{l}\text { - Patients With Positive Non-Screening Cultures for Resistant } \\
\text { Gram positive Isolates identified up to } 2 \text { weeks after } \\
\text { discontinuation of vancomycin }\end{array}$ & 0 & 0 & - & - & - \\
\hline \multicolumn{6}{|l|}{ Vancomycin Use and Dosing } \\
\hline - Final Total Daily Dose, medianº (mg) (Range) & $2000(500-3000)$ & $2000(666-4500)$ & & & 0.46 \\
\hline - Number of Patients With Daily Dose $\geq 3 \mathrm{~g} /$ day $^{c}$ & $4(8 \%)$ & $14(25 \%)$ & 0.27 & $0.08-0.88$ & 0.04 \\
\hline - Initial Steady State Trough $(\mathrm{mg} / \mathrm{L})^{d}$ & $7.13 \pm 2.24$ & $12.20 \pm 5.30$ & & & $<0.0001^{\mathrm{e}}$ \\
\hline - Final Steady State Trough $(\mathrm{mg} / \mathrm{L})^{\mathrm{d}}$ & $7.38 \pm 1.95$ & $16.56 \pm 6.56^{f}$ & & & $<0.0001^{f}$ \\
\hline - Duration of Vancomycin Therapy (days) ${ }^{9}$ & $7 \pm 3$ & $7 \pm 3$ & & & 0.73 \\
\hline • \# Patients Requiring Dose Adjustment & $7(15 \%)$ & $22(40 \%)$ & 0.26 & $0.10-0.67$ & 0.005 \\
\hline - \# of Dose Adjustments Per Patient ${ }^{b}$ & $0.17 \pm 0.43$ & $0.55 \pm 0.79$ & & & $0.02^{f}$ \\
\hline - Time Estimate for Dose Adjustments Per Patient (minutes) ${ }^{b}$ & $9 \pm 23$ & $29 \pm 42$ & & & $0.02^{f}$ \\
\hline \multicolumn{6}{|l|}{ Renal Function Outcomes } \\
\hline • Final Serum Creatinine (mmol/L) & $71 \pm 28$ & $83 \pm 46$ & & & $0.11^{e}$ \\
\hline . \% Change in Serum Creatinine & $-5 \pm 18$ & $3 \pm 30$ & & & $0.13^{e}$ \\
\hline - At Risk for Kidney Injury ${ }^{\text {h }}$ & $0(0 \%)$ & $4(7 \%)$ & 0.12 & $0.01-2.25$ & 0.12 \\
\hline
\end{tabular}

aethicillin Resistant Staphylococcus aureus, Coagulase-negative Staphylococci, or Vancomycin Resistant Enterococci

${ }^{\mathrm{b}}$ For non-normally distributed data, the median was reported rather than the mean \pm standard deviation, with the exception of: \# of Dose Adjustments Per Patient, and Time Estimate for Dose Adjustment Per Patient

'No patients in the low trough group and 2 patients in the high trough group received $\geq 4 \mathrm{~g}$ vancomycin per day

Initial vs. Final steady state trough concentration comparisons within groups: Low trough $p$-value $=0.5607$ (equal SD, unpaired t-test); High trough $p$-value $=$

0.0009 (unequal SD, unpaired t-test Welch Corrected)

eUnequal SD, unpaired t-test Welch Corrected

fDid not pass test for normality, two tailed Mann-Whitney $U$ test used

${ }^{9}$ Patients were included if the intent was to treat for $\leq 14$ days ( 2 patients in the high trough group were treated for 15 days)

$\mathrm{h}_{\geq 50 \%}$ increase from baseline serum creatinine as per RIFLE Criteria [5]

the cohorts. To be adequately powered for a difference in treatment failure of $1 \%$ or $0.5 \%$, a sample size of 17,370 or 68,850 patients per cohort, respectively, would be necessary. Since a difference of $1 \%$ in treatment failure would not be considered clinically important in non-deep seated bacterial infections, this point illustrates the futility for the need to increase the sample size of our study and minimizes any argument that insufficient sample size may have biased against seeing a difference in clinical outcomes between the low and high trough cohorts. To further address the risk of a type II error, we conducted a MCS of 1 million iterations, using a binomial distribution, with the study

Table 3 Univariable and Multivariable Analyses to Identify Factors Associated with Clinical Outcome

\begin{tabular}{|c|c|c|c|c|}
\hline \multirow[t]{2}{*}{ Independent Variables } & \multicolumn{2}{|c|}{$\begin{array}{l}\text { Univariable } \\
\text { (Pearson's Correlation) }\end{array}$} & \multicolumn{2}{|c|}{$\begin{array}{l}\text { Multivariable } \\
\text { (Binary Logistic Regression) }\end{array}$} \\
\hline & Correlation & $P$-value & Odds Ratio & $P$-value \\
\hline Low/High Trough Categorization & -0.003 & 0.973 & - & - \\
\hline Length of Stay at Vancomycin Initiation & -0.259 & 0.008 & 0.988 & 0.554 \\
\hline Heart Failure & -0.247 & 0.012 & 2.513 & 0.319 \\
\hline Pitt Bacteremia Score $\geq 4$ & -0.322 & 0.001 & 4.22 & 0.256 \\
\hline \% Change in Serum Creatinine & -0.201 & 0.042 & 3.139 & 0.538 \\
\hline At Risk for Kidney Injury & -0.378 & $<0.0001$ & 23.606 & 0.163 \\
\hline
\end{tabular}


observed probability of cure (Low trough $=0.88$, High trough $=0.87$ ) and found only a $3 \%$ probability that clinical cure in the high trough cohort could theoretically be $\geq 10 \%$ better than the low trough cohort. This result further supports the validity of the study findings that clinical cure was not significantly different between cohorts. Patients that were treated with vancomycin for less than $48 \mathrm{~h}$ were excluded to minimize the risk of including patients in whom infection was not considered by the physician. Although this exclusion may introduce selection bias due to early mortality, early death attributable to infection would not be anticipated with non-deep seated infections.

Due to the retrospective nature of this study, unidentified confounding factors may exist, and we made assumptions in the definition of steady state levels, as well as the definition of clinical cure and failure. We were unable to calculate the APACHE II score for all ICU patients, as not all patients had arterial blood gases available; we also extrapolated the use of the Pitt Bacteremia score to all hospital ward patients, as there are no other validated measures of severity of illness in this patient population. However, it is reassuring that both groups were well-balanced for all identified demographic, clinical and microbiological factors, including the number of patients with concomitant antibiotics, as well as the number of patients with concomitant antibiotics for the same indication as vancomycin. Only 5\% of patients on vancomycin were eligible for study inclusion, primarily because most patients had deep-seated infections. Therefore, the non-deep seated infection patient population may constitute a small portion of those patients placed on vancomycin, but nevertheless, avoidance of unnecessary increases in vancomycin dosing to provide higher trough concentrations in these patients is an important quality improvement initiative to potentially reduce medication errors associated with frequent dose changes.

\section{Conclusions}

To our knowledge, this is the first study to evaluate clinical and microbiological outcomes associated with trough concentrations in patients with non-deep seated infections treated with short course vancomycin. Our results show an increase in workload with no corresponding clinical benefit, and no signal for increased risk of resistance with vancomycin trough concentrations $\leq 10 \mathrm{mg} / \mathrm{L}$ for short term therapy ( $\leq 14$ days) in non-deep seated infections.

\section{Appendix}

\section{Time Estimate for Vancomycin Dose Adjustment}

- Ordering vancomycin levels: 5 min

- Checking vancomycin level in Electronic Medical Record: 1 min
- Verify correct timing of dosing and levels in Medication Administration Record: 10 min

- Pharmacokinetic calculations: $10 \mathrm{~min}$

- Contact physician for recommendation, obtain telephone order, write telephone order and inform nurse of new medication order: $10 \mathrm{~min}$

- Pharmacist order entry: 2 min

- Preparation of new vancomycin minibag by pharmacy technician: 10 min

- Delivery of new vancomycin minibag to floor: $5 \mathrm{~min}$

Total: $53 \mathrm{~min}$.

\section{Abbreviations}

ACEl: Angiotensin converting enzyme inhibitor; AIDS: Acquired immune deficiency syndrome; APACHE: Acute Physiology and Chronic Health Evaluation; ARB: Angiotensin II receptor blocker; ASA: Acetylsalicylic acid; CNST: Coagulase-negative Staphylococci; CSSTI: Complicated skin and soft tissue infection; EPR: Electronic Patient Record; HCTZ: Hydrochlorothiazide; HDR: Health Data Records; HIV: Human immunodeficiency virus; ICU: Intensive Care Unit; MCS: Monte Carlo Simulation; MRSA: Methicillinresistant Staphylococcus aureus; NSAID: Nonsteroidal anti-inflammatory drug; PPI: Proton pump inhibitor; RIFLE: Risk, Injury, Failure, Loss of kidney function, and End-stage kidney disease; SD: Standard deviation; SHSC: Sunnybrook Health Sciences Centre; SPIRIT: Stewardship Program Integrating Resource Information Technology; SSTI: Skin and soft tissue infection; TNF-a: Tumor necrosis factor alpha; UTI: Urinary tract infection; VRE: Vancomycin-resistant Enterococci

\section{Funding}

This research received no specific grant from any funding agency in the public, commercial or not-for-profit sectors.

\section{Availability of data and materials}

The datasets used and/or analysed during the current study are available from the corresponding author on reasonable request.

\section{Authors' contributions}

SW conceived the project idea, was the project supervisor and is the senior investigator. SW, MW, EM, ME, LP and JA contributed to the design of the study and manuscript development. SW, MW and ME were responsible for development of the data collection tool. MW, EM and ME completed the data collection. SW along with MW and EM analyzed the results. All authors read and approved the final manuscript.

\section{Ethics approval and consent to participate}

This study was approved by the Sunnybrook Health Sciences Centre (SHSC) Research Ethics Board on December 23rd, 2015 (Project Identification Number 497-2015), and was conducted in accordance with the Declaration of Helsinki and national and institutional standards.

\section{Consent for publication}

Not applicable.

\section{Competing interests}

The authors declare that they have no competing interests.

\section{Publisher's Note}

Springer Nature remains neutral with regard to jurisdictional claims in published maps and institutional affiliations.

\section{Author details}

'Department of Pharmacy, Sunnybrook Health Sciences Centre, 2075 Bayview Avenue, Toronto, ON M4N 3M5, Canada. ${ }^{2}$ Leslie Dan Faculty of Pharmacy, University of Toronto, 144 College Street, Toronto, ON M5S 3M2, Canada. ${ }^{3}$ Division of Infectious Diseases, Sunnybrook Health Sciences Centre, 2075 Bayview Avenue, Toronto, ON M4N 3M5, Canada. ${ }^{4}$ Sunnybrook 
Research Institute, Sunnybrook Health Sciences Centre, 2075 Bayview Avenue, Toronto, ON M4N 3M5, Canada. ${ }^{5}$ Department of Medicine, Sunnybrook Health Sciences Centre, 2075 Bayview Avenue, Toronto, ON M4N 3M5, Canada. ${ }^{6}$ Faculty of Medicine, University of Toronto, 1 King's College Circle, Toronto, ON M5S 1A8, Canada. 'Present address: Elaine Martin, Trillium Health Partners, 100 Queensway W, Mississauga, ON L5B 1B8, Canada.

\section{Received: 24 April 2018 Accepted: 11 July 2018}

Published online: 31 July 2018

\section{References}

1. Levine DP. Vancomycin: a history. Clin Infect Dis. 2006:42:S5-12.

2. Rybak MJ. The pharmacokinetic and pharmacodynamics properties of vancomycin. Clin Infect Dis. 2006:42:S35-9.

3. Rybak MJ, Lomaestro BM, Rotschafer JC, Moellering RC, Craig WA, Billeter M, et al. Therapeutic monitoring of vancomycin in adults summary of consensus recommendations from the American Society of Health-System Pharmacists, the Infectious Diseases Society of America, and the Society of Infectious Diseases Pharmacists. Pharmacotherapy. 2009;29:1275-9.

4. Matsumoto K, Takesue $Y$, Ohmagari N, Mochizuki T, Mikamo H, Seki M, et al. Practice guidelines for therapeutic drug monitoring of vancomycin: a consensus review of the Japanese Society of Chemotherapy and the Japanese Society of Therapeutic Drug Monitoring. J Infect Chemother. 2013; 19:365-80

5. Ye Z, Chen Y, Chen K, Zhang X, Du G, He B, et al. Therapeutic drug monitoring of vancomycin: a guideline of the division of therapeutic drug monitoring, Chinese pharmacological society. J Antimicrob Chemother. 2016;71:3020-5.

6. Elligsen $\mathrm{M}$, Walker $\mathrm{SA}$, Simor $\mathrm{AE}$, Daneman N. Prospective audit and feedback of antimicrobial stewardship in critical care: program implementation, experience, and challenges. Can J Hosp Pharm. 2012;65: 31-6.

7. Bellomo R, Ronco C, Kellum J, Mehta R, Palevsky P. Acute dialysis quality initiative workgroup. Acute renal failure - definition, outcome measures, animal models, fluid therapy and information technology needs: the second international consensus conference of the acute dialysis quality initiative (ADQI) group. Crit Care. 2004;8:R204-12.

8. Knaus WA, Draper EA, Wagner DP, Zimmerman JE. APACHE II: a severity of disease classification system. Crit Care Med. 1985;13:818-29.

9. Feldman C, Alanee S, Yu VL, Richards GA, Ortgvist A, Rello J, et al. Severity of illness scoring systems in patients with bacteraemic pneumococcal pneumonia: implications for the intensive care unit care. Clin Microbiol Infect. 2009:15:850-7.

10. Weigelt J, Itani K, Stevens D, Lau W, Dryden M, Knirsch C, et al. Linezolid versus vancomycin in treatment of complicated skin and soft tissue infections. Antimicrob Agents Chemother. 2005;49:2260-6.

11. Stevens DL, Herr D, Lampiris H, Hunt JL, Batts DH, Hafkin B. Linezolid versus vancomycin for the treatment of methicillin-resistant Staphylococcus aureus infections. Clin Infect Dis. 2002;34:1481-90.

12. Itani KM, Dryden MS, Bhattacharyya H, Kunkel MJ, Baruch AM, Weigelt JA. Efficacy and safety of linezolid versus vancomycin for the treatment of complicated skin and soft-tissue infections proven to be caused by methicillin-resistant Staphylococcus aureus. Am J Surg. 2010;199:804-16.

13. Sakoulas G, Eliopoulos GM, Moellering RC, Novick RP, Venkataraman L, Wennersten C, et al. Staphylococcus aureus accessory gene regulator (agr) group II: is there a relationship to the development of intermediate-level glycopeptide resistance? J Infect Dis. 2003;187:929-38.

14. Rose W, Rybak M, Tsuji B, Kaatz G, Sakoulas G. Correlation of vancomycin and daptomycin susceptibility in Staphylococcus aureus in reference to accessory gene regulator (agr) polymorphism and function. J Antimicrob Chemother. 2007:59:1190-3.

15. Tsuji BT, Rybak MJ, Lau KL, Sakoulas G. Evaluation of accessory gene regulator (agr) group and function in the proclivity towards vancomycin intermediate resistance in Staphylococcus aureus. Antimicrob Agents Chemother. 2007:51:1089-91.

16. Zelenitsky S, Alkurdi N, Weber Z, Ariano R, Zhanel G. Preferential emergence of reduced vancomycin susceptibility in health care-associated methicillinresistant Staphylococcus aureus isolates during continuous-infusion vancomycin therapy in an in vitro dynamic model. Antimicrob Agents Chemother. 2011;55:3627-30.
17. Charles PG, Ward PB, Johnson PD, Howden BP, Grayson ML. Clinical features associated with bacteremia due to heterogeneous vancomycin intermediate Staphylococcus aureus. Clin Infect Dis. 2004;38:448-51.

18. Centers for Disease Control and Prevention. Reduced susceptibility of Staphylococcus aureus to vancomycin--Japan. 1996 MMWR Morb Mortal Wkly Rep. 1997:46:624-6.

19. Centers for Disease Control and Prevention. Staphylococcus aureus with reduced susceptibility to vancomycin--United States, 1997. MMWR Morb Mortal Wkly Rep. 1997:46:765-6.

20. Centers for Disease Control and Prevention. Update: Staphylococcus aureus with reduced susceptibility to vancomycin--United States, 1997. MMWR Morb Mortal Wkly Rep. 1997;46:813-5.

21. Smith TL, Pearson ML, Wilcox KR, Cruz C, Lancaster MV, Robinson-Dunn B, et al. Emergence of vancomycin resistance in Staphylococcus aureus. N Engl J Med. 1999:340:493-501.

22. Hageman JC, Pegues DA, Jepson C, Bell RL, Guinan M, Ward KW, et al. Vancomycin-intermediate Staphylococcus aureus in a home health-care patient. Emerg Infect Dis. 2001;7:1023-5.

23. Fridkin SK, Hageman J, McDougal LK, Jarvis WR, Perl TM, Tenover FC, et al. Epidemiological and microbiological characterization of infections caused by Staphylococcus aureus with reduced susceptibility to vancomycin, United States, 1997-2001. Clin Infect Dis. 2003·36:429-39.

\section{Ready to submit your research? Choose BMC and benefit from:}

- fast, convenient online submission

- thorough peer review by experienced researchers in your field

- rapid publication on acceptance

- support for research data, including large and complex data types

- gold Open Access which fosters wider collaboration and increased citations

- maximum visibility for your research: over $100 \mathrm{M}$ website views per year

At $\mathrm{BMC}$, research is always in progress.

Learn more biomedcentral.com/submissions 